\title{
Method of quasilinearization for a nonlocal singular boundary value problem in weighted spaces
}

\author{
Ravi P Agarwall ${ }^{1,2}$, Bashir Ahmad ${ }^{2 *}$ and Ahmed Alsaedi²
}

\section{"Correspondence:}

bashirahmad_qau@yahoo.com

${ }^{2}$ Department of Mathematics,

Faculty of Science, King Abdulaziz

University, P.O. Box 80203, Jeddah,

21589, Saudi Arabia

Full list of author information is

available at the end of the article

\begin{abstract}
This paper studies the existence and uniqueness of solutions for a nonlocal singular boundary value problem of second-order integro-differential equations in weighted spaces. The method of quasilinearization is applied to obtain monotone sequences of approximate solutions converging uniformly and quadratically to a unique solution of the problem at hand. An illustrative example is presented.
\end{abstract}

MSC: $34 \mathrm{~A} 45 ; 34 \mathrm{~B} 16 ; 45 \mathrm{~J} 05$

Keywords: integro-differential equations; nonlocal boundary conditions; quasilinearization; quadratic convergence

\section{Introduction}

Boundary value problems (BVPs) for nonlinear differential equations arise in a variety of areas of applied mathematics, physics and in variational problems of control theory. A point of central importance in the study of nonlinear boundary value problems is to understand how the properties of nonlinearity in a problem influence the nature of the solutions to the boundary value problems. During the last two decades, the theory of singular boundary value problems has also been extensively developed. This is largely due to the fact that the mathematical models in the study of nonlinear phenomena give rise to singular boundary value problems. Examples include energy analysis problems [1, 2], plasma and electric potential theory [3], circular membrane theory [4], membrane response of a spherical cap [5, 6], deformation of membrane cap [7], theory of colloids [8], flow and heat transfer [9], draining flow [10, 11], flow of a gas through porous media [12], Homann flow [13], boundary layer problems [14], pseudoplastic fluids [15], etc. For the theoretical background of singular boundary value problems, we refer the reader to the references $[16,17]$.

Integro-differential equations arise in many engineering and scientific disciplines, often as an approximation to partial differential equations, which represent much of the continuum phenomena. Many forms of these equations are possible. Some of the applications are unsteady aerodynamics and aero-elastic phenomena, visco-elasticity, visco-elastic panel in super sonic gas flow, fluid dynamics, electro-dynamics of complex medium, many models of population growth, polymer rheology, neural network modeling, sandwich system identification, materials with fading memory, mathematical modeling of the diffusion of discrete particles in a turbulent fluid, heat conduction in materials with memory, theory of

@2013 Agarwal et al.; licensee Springer. This is an Open Access article distributed under the terms of the Creative Commons Attribution License (http://creativecommons.org/licenses/by/2.0), which permits unrestricted use, distribution, and reproduction in any medium, provided the original work is properly cited. 
lossless transmission lines, theory of population dynamics, compartmental systems, nuclear reactors and mathematical modeling of hereditary phenomena. Also, the governing equations in the problems of biological sciences, such as spreading of disease by the dispersal of infectious individuals, the reaction-diffusion models in ecology to estimate the speed of invasion etc., are integro-differential equations. Thus, it is important to study singular boundary value problems for nonlinear integro-differential equations. It is worth mentioning that most of the results on singular boundary value problems deal with the existence and uniqueness of the solution of the problems under certain conditions. There are only a few papers which develop some constructive methods for the solution of the nonlinear singular problems.

The study of singular nonlocal boundary value problems for nonlinear differential equations was initiated by Kiguradze and Lomtatidze [18] and Lomtatidze [19, 20]. Since then, more general nonlinear singular nonlocal boundary value problems have been studied extensively. Some results concerning the positive solutions of singular boundary value problems can be found in [21-25] and references therein. A great deal of the work on singular boundary value problems is mainly concerned with the existence of the solution. It is equally important to construct the solution of the problem once its existence is proved.

The monotone iterative technique is one of the efficient analytic methods for solving nonlinear boundary value problems. This technique coupled with the method of upper and lower solutions [26] manifests itself as an effective and flexible mechanism that offers theoretical as well as constructive existence results in a closed set, generated by the lower and upper solutions. In general, the convergence of the sequence of approximate solutions given by the monotone iterative technique is at most linear. To obtain a sequence of approximate solutions converging quadratically, we use the method of quasilinearization (QSL). The origin of the quasilinearization lies in the theory of dynamic programming [27]. In fact, the quasilinearization technique is a variant of Newton's method. This method applies to semilinear equations with convex (concave) nonlinearities and generates a monotone scheme whose iterates converge quadratically to the solution of the problem at hand. In view of its diverse applications, the quasilinearization approach is quite elegant and easier for application algorithms. A detailed description of the QSL method can be found in the monograph [28] and a series of papers [29-48].

Devi and Vatsala [49] discussed the QSL method for second-order singular boundary value problems with solutions in weighted spaces. Eloe [50] developed the QSL method for singular boundary value problems on an unbounded domain. Ramos [51] discussed piecewise quasilinearization techniques for singular boundary value problems. El-Gebeily and O'Regan [52] studied the QSL method for second-order singular nonlinear differential equations with nonlinear boundary conditions. For some existence results on singular initial and boundary value problems, see $[53,54]$. The theoretical background of integrodifferential equations can be found in the text by Lakshmikantham and Rao [55]. To the best of our knowledge, the QSL method has not been discussed for singular nonlocal second-order boundary value problems involving nonlinear integro-differential equations on a bounded domain.

In this paper, we consider the following singular boundary value problem (SBVP):

$$
\begin{cases}-\left(t^{n} u^{\prime}\right)^{\prime}=f\left(t, t^{n-1} u\right)+\int_{0}^{t} K\left(t, s, s^{n-1} u(s)\right) d s, & 0<t<1, n>2, \\ \lim _{t \rightarrow 0^{+}} t^{n-1} u(t)=u_{0}, \quad u(1)=g(u(\sigma)), & 0<\sigma<1,\end{cases}
$$


where $f:[0,1] \times \mathbb{R} \rightarrow \mathbb{R}, K:[0,1] \times[0,1] \times \mathbb{R} \rightarrow \mathbb{R}$, and $g: \mathbb{R} \rightarrow \mathbb{R}$. In the forthcoming analysis, $g(u(\sigma))$ will be written as $g(u)$ for the sake of convenience.

Here we remark that a solution to a singular problem may not lie in the space $C[0,1]$, but it should depend on the singularity in some sense. For example, the solution $u=\left(-u_{0}+\right.$ $\left.u_{1}\right)+u_{0} / t^{2}$ to the singular problem

$$
\left(t^{3} u^{\prime}\right)^{\prime}=0, \quad 0<t<1 ; \quad \lim _{t \rightarrow 0^{+}} t^{2} u(t)=u_{0}, \quad u(1)=u_{1}
$$

$\left(u_{0}, u_{1}\right.$ are constants) is not continuous at $t=0$ but $t^{2} u \in C[0,1]$. Such a situation provides motivation to consider the singular boundary value problems in a weighted Banach space.

\section{Preliminaries and some existence results}

In this section, we follow the terminology introduced in [53].

Definition 2.1 A function $u \in C^{2}(0,1)$ with $t^{n-1} u \in C[0,1], t^{n} u^{\prime} \in C[0,1]$ and $\left(t^{n-1} u\right)^{\prime} \in$ $L^{1}[0,1]$ is a solution of (1.1) if it satisfies singular boundary value problem (1.1).

Remark 2.2 Whenever we say $u(t)$ is a solution of (1.1), it means that $t^{n-1} u(t)$ is a solution of (1.1). Similar terminology will be used for lower and upper solutions.

Throughout the forthcoming analysis, we work in a Banach space of functions defined by

$$
\mathcal{E}=\left\{u \in C^{1}(0,1): t^{n-1} u \in C[0,1], t^{n} u^{\prime} \in C[0,1], t \in[0,1]\right\}
$$

with the norm

$$
\|u\|=\max \left\{\sup _{t \in[0,1]}\left|t^{n-1} u(t)\right|, \sup _{t \in[0,1]}\left|t^{n} u^{\prime}(t)\right|\right\} .
$$

Lemma 2.3 Singular boundary value problem (1.1) is equivalent to the singular integral equation

$$
\begin{aligned}
u(t)= & g(u)-u_{0}\left(1-\frac{1}{t^{n-1}}\right) \\
& +\frac{1-t^{n-1}}{(n-1) t^{n-1}} \int_{0}^{t}\left(f\left(s, s^{n-1} u(s)\right)+\int_{0}^{s} K\left(s, p, p^{n-1} u(p)\right) d p\right) d s \\
& +\frac{1}{(n-1)} \int_{t}^{1} \frac{1-s^{n-1}}{s^{n-1}}\left(f\left(s, s^{n-1} u(s)\right)+\int_{0}^{s} K\left(s, p, p^{n-1} u(p)\right) d p\right) d s .
\end{aligned}
$$

Proof Differentiating (2.1) and rearranging the terms, we have

$$
t^{n} u^{\prime}(t)=-(n-1) u_{0}-\int_{0}^{t}\left(f\left(s, s^{n-1} u(s)\right)+\int_{0}^{s} K\left(s, p, p^{n-1} u(p)\right) d p\right) d s .
$$

Again differentiating, it follows that

$$
-\left(t^{n} u^{\prime}\right)^{\prime}=f\left(t, t^{n-1} u\right)+\int_{0}^{t} K\left(t, s, s^{n-1} u(s)\right) d s .
$$


Multiplying (2.1) by $t^{n-1}$ and taking the limit $t \rightarrow 0^{+}$, we get $\lim _{t \rightarrow 0^{+}} t^{n-1} u(t)=u_{0}$, and $t=1$ in (2.1) yields $u(1)=g(u)$. Thus, $u(t)$ defined by (2.1) satisfies (1.1). A straightforward computation shows that (1.1) implies (2.1). This completes the proof.

Now we prove a general existence principle for singular boundary value problem (1.1). The proof of this principle is based on the Schauder fixed point theorem.

Lemma 2.4 Assume that $f:[0,1] \times \mathbb{R} \rightarrow \mathbb{R}, K:[0,1] \times[0,1] \times \mathbb{R} \rightarrow \mathbb{R}$ and $g: \mathbb{R} \rightarrow \mathbb{R}$ are continuous functions. Furthermore, there exist positive constants $M_{f}, M_{K}, M_{g}$ such that $|f(t, u)| \leq M_{f}$ for $(t, u) \in[0,1] \times \mathbb{R},|K(t, s, u)| \leq M_{K}$ for $(t, s, u) \in[0,1] \times[0,1] \times \mathbb{R}$, and $|g(u)| \leq M_{g}$ for $u \in \mathbb{R}$. Then problem (1.1) has a solution.

Proof We reduce the problem of finding a solution $u \in \mathcal{E}$ of (1.1) to a fixed point problem $u=\mathcal{F} u$, where $\mathcal{F}: \mathcal{E} \rightarrow \mathcal{E}$ is given by

$$
\begin{aligned}
\mathcal{F} u(t)= & g(u)-u_{0}\left(1-\frac{1}{t^{n-1}}\right) \\
& +\frac{1-t^{n-1}}{(n-1) t^{n-1}} \int_{0}^{t}\left(f\left(s, s^{n-1} u(s)\right)+\int_{0}^{s} K\left(s, p, p^{n-1} u(p)\right) d p\right) d s \\
& +\frac{1}{(n-1)} \int_{t}^{1} \frac{1-s^{n-1}}{s^{n-1}}\left(f\left(s, s^{n-1} u(s)\right)+\int_{0}^{s} K\left(s, p, p^{n-1} u(p)\right) d p\right) d s .
\end{aligned}
$$

If $u_{m} \rightarrow u$ in $\mathcal{E}$, then

$$
\begin{aligned}
& \left|t^{n-1} \mathcal{F} u_{m}(t)-t^{n-1} \mathcal{F} u(t)\right| \\
& \leq t^{n-1}\left|g\left(u_{m}\right)-g(u)\right|+\frac{\left(1-t^{n-1}\right)}{(n-1)} \int_{0}^{t}\left(\left|f\left(s, s^{n-1} u_{m}(s)\right)-f\left(s, s^{n-1} u(s)\right)\right|\right. \\
& \left.\quad+\int_{0}^{s}\left|K\left(s, p, p^{n-1} u_{m}(p)\right)-K\left(s, p, p^{n-1} u(p)\right)\right| d p\right) d s \\
& \quad+\frac{t^{n-1}}{(n-1)} \int_{t}^{1}\left(\left|f\left(s, s^{n-1} u_{m}(s)\right)-f\left(s, s^{n-1} u(s)\right)\right|\right. \\
& \left.\quad+\int_{0}^{s}\left|K\left(s, p, p^{n-1} u_{m}(p)\right)-K\left(s, p, p^{n-1} u(p)\right)\right| d p\right) d s,
\end{aligned}
$$

and

$$
\begin{aligned}
& \left|t^{n}\left(\mathcal{F} u_{m}\right)^{\prime}(t)-t^{n}(\mathcal{F} u)^{\prime}(t)\right| \\
& \quad \leq \int_{0}^{t}\left(\left|f\left(s, s^{n-1} u_{m}(s)\right)-f\left(s, s^{n-1} u(s)\right)\right|\right. \\
& \left.\quad+\int_{0}^{s}\left|K\left(s, p, p^{n-1} u_{m}(p)\right)-K\left(s, p, p^{n-1} u(p)\right)\right| d p\right) d s .
\end{aligned}
$$

In view of the continuity of $f, K, g$, it follows that $\mathcal{F} u_{m} \rightarrow \mathcal{F} u$ in $\mathcal{E}$ and so $\mathcal{F}: \mathcal{E} \rightarrow \mathcal{E}$ is continuous. 
Now, for $r, t \in[0,1]$ with $r<t$, we have

$$
\begin{aligned}
\left|t^{n-1} \mathcal{F} u(t)-r^{n-1} \mathcal{F} u(r)\right| & \\
\leq & \left|t^{n-1}-r^{n-1}\right|\left(M_{g}+\left|u_{0}\right|\right)+\frac{1}{(n-1)}\left|t^{n-1}-r^{n-1}\right| \int_{0}^{r}\left(M_{f}+\int_{0}^{s} M_{K} d p\right) d s \\
& +\frac{\left(1-t^{n-1}\right)}{(n-1)} \int_{r}^{t}\left(M_{f}+\int_{0}^{s} M_{K} d p\right) d s \\
& +\frac{1}{(n-1)}\left|t^{n-1}-r^{n-1}\right| \int_{t}^{1} \frac{1-s^{n-1}}{s^{n-1}}\left(M_{f}+\int_{0}^{s} M_{K} d p\right) d s \\
& +\frac{\left(1-t^{n-1}\right)}{(n-1)} \int_{r}^{t}\left(M_{f}+\int_{0}^{s} M_{K} d p\right) d s \\
& +\frac{r^{n-1}}{(n-1)} \int_{r}^{t} \frac{1-s^{n-1}}{s^{n-1}}\left(M_{f}+\int_{0}^{s} M_{K} d p\right) d s
\end{aligned}
$$

and

$$
\left|r^{n}(\mathcal{F} u)^{\prime}(r)-t^{n}(\mathcal{F} u)^{\prime}(t)\right| \leq \int_{r}^{t}\left(M_{f}+\int_{0}^{s} M_{K} d p\right) d s,
$$

which are independent of $u$. So $\mathcal{F}: \mathcal{E} \rightarrow \mathcal{E}$ is relatively compact. Hence, by the ArzelaAscoli theorem, $\mathcal{F}$ is compact on $\mathcal{E}$. Thus, by Schauder's fixed point theorem, $\mathcal{F}$ has a fixed point in $\mathcal{E}$. This completes the proof.

Definition 2.5 A function $\beta \in C^{2}(0,1)$ such that $t^{n-1} \beta \in C[0,1], t^{n} \beta^{\prime} \in C[0,1],\left(t^{n-1} \beta\right)^{\prime} \in$ $L^{1}[0,1]$ is called an upper solution for $(1.1)$ if

$$
\begin{aligned}
& -\left(t^{n} \beta^{\prime}\right)^{\prime} \geq f\left(t, t^{n-1} \beta\right)+\int_{0}^{t} K\left(t, s, s^{n-1} \beta(s)\right) d s, \quad 0<t<1, \\
& \lim _{t \rightarrow 0^{+}} t^{n-1} \beta(t) \geq u_{0}, \quad \beta(1) \geq g(\beta) .
\end{aligned}
$$

Similarly, a function $\alpha \in C^{2}(0,1)$ such that $t^{n-1} \alpha \in C[0,1], t^{n} \alpha^{\prime} \in C[0,1],\left(t^{n-1} \alpha\right)^{\prime} \in L^{1}[0,1]$ is called a lower solution for (1.1) if

$$
\begin{aligned}
& -\left(t^{n} \alpha^{\prime}\right)^{\prime} \leq f\left(t, t^{n-1} \alpha\right)+\int_{0}^{t} K\left(t, s, s^{n-1} \alpha(s)\right) d s, \quad 0<t<1, \\
& \lim _{t \rightarrow 0^{+}} t^{n-1} \alpha(t) \leq u_{0}, \quad \alpha(1) \leq g(\alpha) .
\end{aligned}
$$

Lemma 2.6 Assume that $f:[0,1] \times \mathbb{R} \rightarrow \mathbb{R}$ and $K:[0,1] \times[0,1] \times \mathbb{R} \rightarrow \mathbb{R}$ are continuous functions, and $g: \mathbb{R} \rightarrow \mathbb{R}$ is a Lipschitz function. Suppose that there exist an upper solution $\beta$ and a lower solution $\alpha$ for (1.1) such that $\alpha \leq \beta$ on $(0,1)$, and $\lim _{t \rightarrow 0^{+}} t^{n-1} \alpha(t) \leq u_{0} \leq$ $\lim _{t \rightarrow 0^{+}} t^{n-1} \beta(t)$. Then (1.1) has a solution $u$ satisfying the relation $t^{n-1} \alpha(t) \leq t^{n-1} u(t) \leq$ $t^{n-1} \beta(t)$ for $t \in[0,1]$.

Proof Consider the modified problem

$$
\begin{array}{ll}
-\left(t^{n} u^{\prime}\right)^{\prime}=F\left(t, t^{n-1} u\right), & 0<t<1, \\
\lim _{t \rightarrow 0^{+}} t^{n-1} u(t)=u_{0}, & u(1)=g(u),
\end{array}
$$


where

$$
F(t, v)= \begin{cases}f\left(t, t^{n-1} \beta\right)+\int_{0}^{t} K\left(t, s, s^{n-1} \beta(s)\right) d s-\left(v-t^{n-1} \beta\right) & \text { if } v \geq t^{n-1} \beta(t), \\ f(t, v)+\int_{0}^{t} K(t, s, v) d s & \text { if } t^{n-1} \alpha(t) \leq v \leq t^{n-1} \beta(t), \\ f\left(t, t^{n-1} \alpha\right)+\int_{0}^{t} K\left(t, s, s^{n-1} \alpha(s)\right) d s-\left(v-t^{n-1} \alpha\right) & \text { if } v \leq t^{n-1} \alpha(t) .\end{cases}
$$

By Lemma 2.4, problem (2.2) has a solution $u$. Let us claim that

$$
t^{n-1} \alpha(t) \leq t^{n-1} u(t) \leq t^{n-1} \beta(t), \quad t \in[0,1] .
$$

If (2.3) is true, then we are done, that is, $u$ is a solution of (1.1). On the contrary, assume that $t^{n-1} u(t) \not \leq t^{n-1} \beta(t)$ for $t \in[0,1]$. Then there exists $t_{0} \in(0,1)$ such that $\left(t^{n-1} u(t)-\right.$ $\left.t^{n-1} \beta(t)\right)$ has a positive maximum at $t_{0}$. Thus, $\left(t^{n-1} u(t)-t^{n-1} \beta(t)\right)^{\prime}\left(t_{0}\right)=0$ and $\left(t^{n-1} u(t)-\right.$ $\left.t^{n-1} \beta(t)\right)^{\prime \prime}\left(t_{0}\right) \leq 0$. On the other hand,

$$
\begin{aligned}
\left(t^{n-1} u(t)-t^{n-1} \beta(t)\right)^{\prime \prime}\left(t_{0}\right) \\
=\frac{1}{t_{0}}\left(t_{0}^{n} u^{\prime}\left(t_{0}\right)\right)^{\prime}+(n-2) t_{0}^{n-2} u^{\prime}\left(t_{0}\right)+(n-1)(n-2) t_{0}^{n-3} u\left(t_{0}\right) \\
\quad-\frac{1}{t_{0}}\left(t_{0}^{n} \beta^{\prime}\left(t_{0}\right)\right)^{\prime}-(n-2) t_{0}^{n-2} \beta^{\prime}\left(t_{0}\right)-(n-1)(n-2) t_{0}^{n-3} \beta\left(t_{0}\right) \\
=\frac{1}{t_{0}}\left(t_{0}^{n} u^{\prime}\left(t_{0}\right)\right)^{\prime}-\frac{1}{t_{0}}\left(t_{0}^{n} \beta^{\prime}\left(t_{0}\right)\right)^{\prime} \\
\geq \\
\geq \frac{1}{t_{0}}\left(t_{0}^{n} u^{\prime}\left(t_{0}\right)\right)^{\prime}+\frac{1}{t_{0}}\left(f\left(t_{0}, t_{0}^{n-1} \beta\left(t_{0}\right)\right)+\int_{0}^{t_{0}} K\left(t_{0}, s, s^{n-1} \beta(s)\right) d s\right) \\
=\frac{1}{t_{0}}\left(t_{0}^{n-1} u\left(t_{0}\right)-t_{0}^{n-1} \beta\left(t_{0}\right)\right)>0,
\end{aligned}
$$

which is a contradiction. Hence $t^{n-1} u(t) \leq t^{n-1} \beta(t)$ for $t \in(0,1)$. Similarly, it can be shown that $t^{n-1} \alpha(t) \leq t^{n-1} u(t)$ for $t \in(0,1)$. In view of assumptions that $g$ is a Lipschitz function and $\alpha \leq \beta$ on $(0,1)$, it follows that $\alpha(1) \leq \beta(1)$. Thus we conclude that

$$
t^{n-1} \alpha(t) \leq t^{n-1} u(t) \leq t^{n-1} \beta(t) \quad \text { for } t \in[0,1] .
$$

This completes the proof.

Lemma 2.7 Let $\alpha, \beta \in \mathcal{E}$ be lower and upper solutions of (1.1) respectively with

$$
\lim _{t \rightarrow 0^{+}} t^{n-1} \alpha(t) \leq u_{0} \leq \lim _{t \rightarrow 0^{+}} t^{n-1} \beta(t) .
$$

Further, suppose that $f:[0,1] \times \mathbb{R} \rightarrow \mathbb{R}, K:[0,1] \times[0,1] \times \mathbb{R} \rightarrow \mathbb{R}$ and $g: \mathbb{R} \rightarrow \mathbb{R}$ are continuous functions and satisfy

$$
\begin{aligned}
& f\left(t, t^{n-1} x\right)-f\left(t, t^{n-1} y\right) \leq-L_{1}(x-y), \quad L_{1}>0, \\
& K\left(t, s, s^{n-1} x\right)-K\left(t, s, s^{n-1} y\right) \leq-L_{2}(x-y), \quad L_{2}>0, \\
& g(x)-g(y) \leq L_{3}(x-y), \quad 0<L_{3}<1,
\end{aligned}
$$

whenever $x \geq y$. Then $t^{n-1} \alpha(t) \leq t^{n-1} \beta(t)$ on $[0,1]$. 
Proof For the sake of contradiction, suppose that the conclusion does not hold, that is, $t^{n-1} \alpha(t)>t^{n-1} \beta(t)$ at some $t \in(0,1]$. Then, by continuity, there exists a local maximum at $t_{0} \in(0,1)$. Thus, at $t=t_{0}$, we have

$$
\begin{aligned}
& \left(t^{n-1}(\alpha-\beta)\right)^{\prime}=0, \\
& \left(t^{n-1}(\alpha-\beta)\right)^{\prime \prime} \leq 0 .
\end{aligned}
$$

In view of (2.4), (2.5) takes the form

$$
\begin{aligned}
0 \geq & {\left[t^{n-1}(\alpha-\beta)\right]^{\prime \prime} } \\
= & {\left[(n-1) t^{n-2}(\alpha-\beta)+t^{n-1}\left(\alpha^{\prime}-\beta^{\prime}\right)\right]^{\prime} } \\
= & (n-1)(n-2) t^{n-3}(\alpha-\beta)+(n-1) t^{n-2}\left(\alpha^{\prime}-\beta^{\prime}\right) \\
& +(n-1) t^{n-2}\left(\alpha^{\prime}-\beta^{\prime}\right)+t^{n-1}\left(\alpha^{\prime \prime}-\beta^{\prime \prime}\right) \\
= & (n-1)(n-2) t^{n-3}(\alpha-\beta)+2(n-1) t^{n-2}\left(\alpha^{\prime}-\beta^{\prime}\right)+t^{n-1}\left(\alpha^{\prime \prime}-\beta^{\prime \prime}\right) \\
= & (n-1)(n-2) t^{n-3}(\alpha-\beta)+(2 n-2) t^{n-2}\left(\alpha^{\prime}-\beta^{\prime}\right)+t^{n-1}\left(\alpha^{\prime \prime}-\beta^{\prime \prime}\right) \\
= & (n-1)(n-2) t^{n-3}(\alpha-\beta)+(n-2) t^{n-2}\left(\alpha^{\prime}-\beta^{\prime}\right) \\
& +n t^{n-2} \alpha^{\prime}-n t^{n-2} \beta^{\prime}+t^{n-1}\left(\alpha^{\prime \prime}-\beta^{\prime \prime}\right) \\
= & \frac{n-2}{t}\left[(n-1) t^{n-2}(\alpha-\beta)+t^{n-1}\left(\alpha^{\prime}-\beta^{\prime}\right)\right] \\
& +n t^{n-2} \alpha^{\prime}-n t^{n-2} \beta^{\prime}+t^{n-1} \alpha^{\prime \prime}-t^{n-1} \beta^{\prime \prime} \\
= & \frac{n-2}{t}\left[t^{n-1}(\alpha-\beta)\right]^{\prime}+n t^{n-2} \alpha^{\prime}+t^{n-1} \alpha^{\prime \prime}-n t^{n-2} \beta^{\prime}-t^{n-1} \beta^{\prime \prime} \\
= & \frac{n-2}{t}(0)+n t^{n-2} \alpha^{\prime}+t^{n-1} \alpha^{\prime \prime}-n t^{n-2} \beta^{\prime}-t^{n-1} \beta^{\prime \prime} \\
= & \frac{1}{t}\left(t^{n} \alpha^{\prime}\right)^{\prime}-\frac{1}{t}\left(t^{n} \beta^{\prime}\right)^{\prime},
\end{aligned}
$$

which can alternatively be written as $-t^{-1}\left(t^{n} \alpha^{\prime}\right)^{\prime} \geq-t^{-1}\left(t^{n} \beta^{\prime}\right)^{\prime}$ at $t=t_{0} \in(0,1)$. Using the definition of lower and upper solutions together with (2.5), we obtain

$$
\begin{aligned}
& f\left(t_{0}, t_{0}^{n-1} \alpha\right)+\int_{0}^{t_{0}} K\left(t_{0}, s, s^{n-1} \alpha(s)\right) d s \\
& \geq-\left(t_{0}^{n} \alpha^{\prime}\right)^{\prime} \geq-\left(t_{0}^{n} \beta^{\prime}\right)^{\prime} \\
& \geq f\left(t, t_{0}^{n-1} \beta\right)+\int_{0}^{t_{0}} K\left(t_{0}, s, s^{n-1} \beta(s)\right) d s,
\end{aligned}
$$

which yields a contradiction:

$$
\begin{aligned}
0 & \leq f\left(t_{0}, t_{0}^{n-1} \alpha\right)-f\left(t, t_{0}^{n-1} \beta\right)+\int_{0}^{t_{0}}\left[K\left(t_{0}, s, s^{n-1} \alpha(s)\right)-K\left(t_{0}, s, s^{n-1} \beta(s)\right)\right] d s \\
& \leq-\left(L_{1}+t_{0} L_{2}\right) t_{0}^{n-1}(\alpha-\beta)<0 .
\end{aligned}
$$


Hence we obtain $t^{n-1} \alpha(t) \leq t^{n-1} \beta(t)$ on $(0,1)$. As $0<\sigma<1$, therefore, in view of the foregoing arguments, at $t_{0}=1$, we have

$$
\begin{aligned}
0 & <\alpha(1)-\beta(1) \leq g(\alpha)-g(\beta) \\
& \leq \frac{L_{3}}{\sigma^{n-1}}\left(\sigma^{n-1} \alpha(\sigma)-\sigma^{n-1} \beta(\sigma)\right) \leq 0, \quad 0<\sigma<1,
\end{aligned}
$$

which is a contradiction. This completes the proof.

\section{Main result}

\section{Theorem 3.1 Assume that}

$\left(\mathrm{A}_{1}\right) \alpha_{0}, \beta_{0} \in \mathcal{E}$ are lower and upper solutions of (1.1) respectively such that $t^{n-1} \alpha_{0}(t) \leq$ $t^{n-1} \beta_{0}(t)$ for $t \in(0,1]$ with $\lim _{t \rightarrow 0^{+}} t^{n-1} \alpha_{0}(t) \leq u_{0} \leq \lim _{t \rightarrow 0^{+}} t^{n-1} \beta_{0}(t) ;$

$\left(\mathrm{A}_{2}\right) f(t, v)$ is convex, that is, $f_{v v}(t, v)$ exists, continuous with $f_{v v}(t, v) \geq 0$ for each $(t, v) \in$ $(0,1] \times \mathbb{R}$, and $f_{v}\left(t, t^{n-1} u\right)<0$, where $t^{n-1} \alpha_{0} \leq v \leq t^{n-1} \beta_{0} ;$

$\left(\mathrm{A}_{3}\right) K(t, s, v)$ is convex, that is, $K_{v v}(t, s, v)$ exists, continuous with $K_{v v}(t, s, v) \geq 0$ for each $(t, s, v) \in(0,1] \times(0,1] \times \mathbb{R}$, and $K_{v}\left(t, s, s^{n-1} u\right)<0$, where $t^{n-1} \alpha_{0} \leq v \leq t^{n-1} \beta_{0} ;$

$\left(\mathrm{A}_{4}\right) g$ is continuous on $\mathbb{R}$ such that $g^{\prime}, g^{\prime \prime}$ exist and $0 \leq g^{\prime}<1, g^{\prime \prime} \leq 0$.

Then there exist monotone sequences $\left\{t^{n-1} \alpha_{k}(t)\right\}$ and $\left\{t^{n-1} \beta_{k}(t)\right\}$ that converge uniformly and quadratically in the space of continuous functions on $[0,1]$ to a unique solution $t^{n-1} u(t)$ of (1.1).

Proof Using the generalized mean value theorem together with $\left(\mathrm{A}_{2}\right),\left(\mathrm{A}_{3}\right)$, and $\left(\mathrm{A}_{4}\right)$, we obtain

$$
\begin{aligned}
& f\left(t, t^{n-1} u\right) \geq f\left(t, t^{n-1} w\right)+f_{v}\left(t, t^{n-1} w\right)(u-w) t^{n-1}, \quad u \geq w, \\
& K\left(t, s, s^{n-1} u\right) \geq K\left(t, s, s^{n-1} w\right)+k_{v}\left(t, s, s^{n-1} w\right)(u-w) s^{n-1}, \quad u \geq w, \\
& g(u) \leq g(w)+g^{\prime}(w)(u-w) .
\end{aligned}
$$

Now, we set

$$
\begin{aligned}
& F\left(t, t^{n-1} \alpha_{1} ; \alpha_{0}\right)=f\left(t, t^{n-1} \alpha_{0}\right)+f_{v}\left(t, t^{n-1} \alpha_{0}\right)\left(\alpha_{1}-\alpha_{0}\right) t^{n-1}, \\
& \bar{F}\left(t, t^{n-1} \beta_{1} ; \alpha_{0}, \beta_{0}\right)=f\left(t, t^{n-1} \beta_{0}\right)+f_{v}\left(t, t^{n-1} \alpha_{0}\right)\left(\beta_{1}-\beta_{0}\right) t^{n-1}, \\
& K^{*}\left(t, s, s^{n-1} \alpha_{1} ; \alpha_{0}\right)=K\left(t, s, s^{n-1} \alpha_{0}\right)+K_{v}\left(t, s, s^{n-1} \alpha_{0}\right)\left(\alpha_{1}-\alpha_{0}\right) s^{n-1}, \\
& K^{* *}\left(t, s, s^{n-1} \beta_{1} ; \alpha_{0}, \beta_{0}\right)=K\left(t, s, s^{n-1} \beta_{0}\right)+K_{v}\left(t, s, s^{n-1} \alpha_{0}\right)\left(\beta_{1}-\beta_{0}\right) s^{n-1}, \\
& h_{1}\left(\alpha_{1} ; \alpha_{0}, \beta_{0}\right)=g\left(\alpha_{0}\right)+g^{\prime}\left(\beta_{0}\right)\left(\alpha_{1}-\alpha_{0}\right), \\
& h_{2}\left(\beta_{1} ; \beta_{0}\right)=g\left(\beta_{0}\right)+g^{\prime}\left(\beta_{0}\right)\left(\beta_{1}-\beta_{0}\right) .
\end{aligned}
$$

Consider the singular BVP

$$
\left\{\begin{array}{l}
-\left(t^{n} \alpha_{1}^{\prime}\right)^{\prime}=F\left(t, t^{n-1} \alpha_{1} ; \alpha_{0}\right)+\int_{0}^{t} K^{*}\left(t, s, s^{n-1} \alpha_{1} ; \alpha_{0}\right) d s, \quad 0<t<1, \\
\lim _{t \rightarrow 0^{+}} t^{n-1} \alpha_{1}(t)=u_{0}, \quad \alpha_{1}(1)=h_{1}\left(\alpha_{1} ; \alpha_{0}, \beta_{0}\right) .
\end{array}\right.
$$


Using assumption $\left(\mathrm{A}_{1}\right),(3.1)$ and (3.2), we obtain

$$
\begin{aligned}
-\left(t^{n} \alpha_{0}^{\prime}\right)^{\prime} & \leq f\left(t, t^{n-1} \alpha_{0}\right)+\int_{0}^{t} K\left(t, s, s^{n-1} \alpha_{0}(s)\right) d s \\
& =F\left(t, t^{n-1} \alpha_{0} ; \alpha_{0}\right)+\int_{0}^{t} K^{*}\left(t, s, s^{n-1} \alpha_{0} ; \alpha_{0}\right) d s
\end{aligned}
$$

and

$$
\begin{aligned}
-\left(t^{n} \beta_{0}^{\prime}\right)^{\prime} \geq & f\left(t, t^{n-1} \beta_{0}\right)+\int_{0}^{t} K\left(t, s, s^{n-1} \beta_{0}\right) d s \\
\geq & f\left(t, t^{n-1} \alpha_{0}\right)+f_{v}\left(t, t^{n-1} \alpha_{0}\right)\left(\beta_{0}-\alpha_{0}\right) t^{n-1} \\
& \quad+\int_{0}^{t}\left[K\left(t, s, s^{n-1} \alpha_{0}\right)+k_{\nu}\left(t, s, s^{n-1} \alpha_{0}\right)\left(\beta_{0}-\alpha_{0}\right) s^{n-1}\right] d s \\
= & F\left(t, t^{n-1} \beta_{0} ; \alpha_{0}\right)+\int_{0}^{t} K^{*}\left(t, s, s^{n-1} \beta_{0} ; \alpha_{0}\right) d s .
\end{aligned}
$$

Further, we note that $\alpha_{0}(1) \leq g\left(\alpha_{0}\right)=h_{1}\left(\alpha_{0} ; \alpha_{0}, \beta_{0}\right)$ and using assumption $\left(\mathrm{A}_{4}\right)$, for $c_{0} \in$ $\left(\alpha_{0}, \beta_{0}\right)$, we find that

$$
\begin{aligned}
g\left(\beta_{0}\right)-h_{1}\left(\beta_{0} ; \alpha_{0}, \beta_{0}\right) & =g\left(\beta_{0}\right)-g\left(\alpha_{0}\right)-g^{\prime}\left(\beta_{0}\right)\left(\beta_{0}-\alpha_{0}\right) \\
& =\left[g^{\prime}\left(c_{0}\right)-g^{\prime}\left(\beta_{0}\right)\right]\left(\beta_{0}-\alpha_{0}\right) \geq 0,
\end{aligned}
$$

which implies that $\beta_{0}(1) \geq g\left(\beta_{0}\right) \geq h_{1}\left(\beta_{0} ; \alpha_{0}, \beta_{0}\right)$. Thus, it follows that $\alpha_{0}$ and $\beta_{0}$ are respectively lower and upper solutions of (3.4). Since $h_{i}^{\prime}=g^{\prime}(i=1,2)$, therefore it follows by Lemmas 2.6 and 2.7 that there exists a unique solution $\alpha_{1}$ of (3.4) satisfying

$$
t^{n-1} \alpha_{0}(t) \leq t^{n-1} \alpha_{1}(t) \leq t^{n-1} \beta_{0}(t), \quad t \in[0,1] .
$$

Observe that the hypotheses of Lemma 2.7 hold in view of the conditions $f_{v}<0, K_{v}<0$, and $0 \leq g^{\prime}<1$, demanded in $\left(\mathrm{A}_{2}\right),\left(\mathrm{A}_{3}\right)$, and $\left(\mathrm{A}_{4}\right)$.

Next, consider the singular BVP

$$
\left\{\begin{array}{l}
-\left(t^{n} \beta_{1}^{\prime}\right)^{\prime}=\bar{F}\left(t, t^{n-1} \beta_{1} ; \alpha_{0}, \beta_{0}\right)+\int_{0}^{t} K^{* *}\left(t, s, s^{n-1} \beta_{1} ; \alpha_{0}, \beta_{0}\right) d s, \quad 0<t<1, \\
\lim _{t \rightarrow 0^{+}} t^{n-1} \beta_{1}(t)=u_{0}, \quad \beta_{1}(1)=h_{2}\left(\beta_{1} ; \beta_{0}\right) .
\end{array}\right.
$$

Using the definition of the lower and upper solutions, (3.1) and (3.2), we get

$$
\begin{aligned}
-\left(t^{n} \alpha_{0}^{\prime}\right)^{\prime} \leq & f\left(t, t^{n-1} \alpha_{0}\right)+\int_{0}^{t} K\left(t, s, s^{n-1} \alpha_{0}(s)\right) d s \\
\leq & f\left(t, t^{n-1} \beta_{0}\right)+f_{v}\left(t, t^{n-1} \alpha_{0}\right)\left(\alpha_{0}-\beta_{0}\right) t^{n-1} \\
& +\int_{0}^{t}\left[K\left(t, s, s^{n-1} \beta_{0}\right)+K_{v}\left(t, s, s^{n-1} \alpha_{0}\right)\left(\alpha_{0}-\beta_{0}\right) s^{n-1}\right] d s \\
= & \bar{F}\left(t, t^{n-1} \alpha_{0} ; \alpha_{0}, \beta_{0}\right)+\int_{0}^{t} K^{* *}\left(t, s, s^{n-1} \alpha_{0} ; \alpha_{0}, \beta_{0}\right) d s,
\end{aligned}
$$




$$
\begin{aligned}
-\left(t^{n} \beta_{0}^{\prime}\right)^{\prime} & \geq f\left(t, t^{n-1} \beta_{0}\right)+\int_{0}^{t} K\left(t, s, s^{n-1} \beta_{0}\right) d s \\
& =\bar{F}\left(t, t^{n-1} \beta_{0} ; \alpha_{0}, \beta_{0}\right)+\int_{0}^{t} K^{* *}\left(t, s, s^{n-1} \beta_{0} ; \alpha_{0}, \beta_{0}\right) d s .
\end{aligned}
$$

By assumption $\left(\mathrm{A}_{4}\right)$, we have $\alpha_{0}(1) \leq h_{2}\left(\alpha_{0} ; \beta_{0}\right)$ as

$$
\begin{aligned}
g\left(\alpha_{0}\right)-h_{2}\left(\alpha_{0} ; \beta_{0}\right) & =g\left(\alpha_{0}\right)-g\left(\beta_{0}\right)+g^{\prime}\left(\beta_{0}\right)\left(\beta_{0}-\alpha_{0}\right) \\
& =\left[-g^{\prime}\left(c_{1}\right)+g^{\prime}\left(\beta_{0}\right)\right]\left(\beta_{0}-\alpha_{0}\right) \leq 0, \quad c_{1} \in\left(\alpha_{0}, \beta_{0}\right),
\end{aligned}
$$

and $\beta_{0}(1) \geq g\left(\beta_{0}\right)=h_{2}\left(\beta_{0} ; \beta_{0}\right)$. In view of the above inequalities, we find that $\alpha_{0}$ and $\beta_{0}$ are respectively lower and upper solutions of (3.5). Therefore it follows by Lemmas 2.6 and 2.7 that there exists a unique solution $\beta_{1}$ of (3.5) satisfying

$$
t^{n-1} \alpha_{0}(t) \leq t^{n-1} \beta_{1}(t) \leq t^{n-1} \beta_{0}(t), \quad t \in[0,1] .
$$

Now we show that $t^{n-1} \alpha_{1}(t) \leq t^{n-1} \beta_{1}(t)$. Using (3.1), (3.2), and assumption ( $\left.\mathrm{A}_{1}\right)$, we obtain

$$
\begin{aligned}
-\left(t^{n} \alpha_{1}^{\prime}\right)^{\prime}= & F\left(t, t^{n-1} \alpha_{1} ; \alpha_{0}\right)+\int_{0}^{t} K^{*}\left(t, s, s^{n-1} \alpha_{1} ; \alpha_{0}\right) d s \\
= & f\left(t, t^{n-1} \alpha_{0}\right)+f_{v}\left(t, t^{n-1} \alpha_{0}\right)\left(\alpha_{1}-\alpha_{0}\right) t^{n-1} \\
& +\int_{0}^{t}\left[K\left(t, s, s^{n-1} \alpha_{0}\right)+K_{v}\left(t, s, s^{n-1} \alpha_{0}\right)\left(\alpha_{1}-\alpha_{0}\right) s^{n-1}\right] d s \\
\leq & f\left(t, t^{n-1} \beta_{0}\right)+f_{v}\left(t, t^{n-1} \alpha_{0}\right)\left(\alpha_{0}-\beta_{0}\right) t^{n-1}+f_{v}\left(t, t^{n-1} \alpha_{0}\right)\left(\alpha_{1}-\alpha_{0}\right) t^{n-1} \\
& +\int_{0}^{t}\left[K\left(t, s, s^{n-1} \beta_{0}\right)+K_{v}\left(t, s, s^{n-1} \alpha_{0}\right)\left(\alpha_{0}-\beta_{0}\right) s^{n-1}\right. \\
& \left.+K_{v}\left(t, s, s^{n-1} \alpha_{0}\right)\left(\alpha_{1}-\alpha_{0}\right) s^{n-1}\right] d s \\
= & f\left(t, t^{n-1} \beta_{0}\right)+f_{v}\left(t, t^{n-1} \alpha_{0}\right)\left(\alpha_{1}-\beta_{0}\right) t^{n-1} \\
& +\int_{0}^{t}\left[K\left(t, s, s^{n-1} \beta_{0}\right)+K_{v}\left(t, s, s^{n-1} \alpha_{0}\right)\left(\alpha_{1}-\beta_{0}\right) s^{n-1}\right] d s \\
= & \bar{F}\left(t, t^{n-1} \alpha_{1} ; \alpha_{0}, \beta_{0}\right)+\int_{0}^{t} K^{* *}\left(t, s, s^{n-1} \alpha_{1} ; \alpha_{0}, \beta_{0}\right) d s
\end{aligned}
$$

and in view of $\left(\mathrm{A}_{4}\right)$, we get $g\left(\alpha_{1}\right)-h_{2}\left(\alpha_{1} ; \beta_{0}\right) \leq 0$, which implies that $\alpha_{1}(1) \leq h_{2}\left(\alpha_{1} ; \beta_{0}\right)$. Clearly, the above inequalities and singular boundary value problem (3.5) satisfy the hypotheses of Lemma 2.7. Therefore, by the conclusion of Lemma 2.7, we have $t^{n-1} \alpha_{1}(t) \leq$ $t^{n-1} \beta_{1}(t), t \in[0,1]$. Consequently, we obtain

$$
t^{n-1} \alpha_{0}(t) \leq t^{n-1} \alpha_{1}(t) \leq t^{n-1} \beta_{1}(t) \leq t^{n-1} \beta_{0}(t), \quad t \in[0,1] .
$$

As a next step, we prove that

$$
t^{n-1} \alpha_{k}(t) \leq t^{n-1} \alpha_{k+1}(t) \leq t^{n-1} \beta_{k+1}(t) \leq t^{n-1} \beta_{k}(t), \quad t \in[0,1]
$$


for $k>1$. For that, we consider the following SBVP:

$$
\left\{\begin{array}{l}
-\left(t^{n} \alpha_{k+1}^{\prime}\right)^{\prime}=F\left(t, t^{n-1} \alpha_{k+1} ; \alpha_{k}\right)+\int_{0}^{t} K^{*}\left(t, s, s^{n-1} \alpha_{k+1} ; \alpha_{k}\right) d s, \quad 0<t<1, \\
\lim _{t \rightarrow 0^{+}} t^{n-1} \alpha_{k+1}(t)=u_{0}, \quad \alpha_{k+1}(1)=h_{1}\left(\alpha_{k+1} ; \alpha_{k}, \beta_{0}\right) .
\end{array}\right.
$$

Using (3.1), (3.2), (3.3), and the inequality $t^{n-1} \alpha_{k-1} \leq t^{n-1} \alpha_{k}$, we get

$$
\begin{aligned}
&-\left(t^{n} \alpha_{k}^{\prime}\right)^{\prime}= F\left(t, t^{n-1} \alpha_{k} ; \alpha_{k-1}\right)+\int_{0}^{t} K^{*}\left(t, s, s^{n-1} \alpha_{k} ; \alpha_{k-1}\right) d s \\
&= f\left(t, t^{n-1} \alpha_{k-1}\right)+f_{v}\left(t, t^{n-1} \alpha_{k-1}\right)\left(\alpha_{k}-\alpha_{k-1}\right) t^{n-1} \\
&+\int_{0}^{t}\left[K\left(t, s, s^{n-1} \alpha_{k-1}\right)+K_{v}\left(t, s, s^{n-1} \alpha_{k-1}\right)\left(\alpha_{k}-\alpha_{k-1}\right) s^{n-1}\right] d s \\
& \leq f\left(t, t^{n-1} \alpha_{k}\right)-f_{v}\left(t, t^{n-1} \alpha_{k-1}\right)\left(\alpha_{k}-\alpha_{k-1}\right) t^{n-1} \\
&+f_{v}\left(t, t^{n-1} \alpha_{k-1}\right)\left(\alpha_{k}-\alpha_{k-1}\right) t^{n-1} \\
&+\int_{0}^{t}\left[K\left(t, s, s^{n-1} \alpha_{k}\right)-K_{v}\left(t, s, s^{n-1} \alpha_{k-1}\right)\left(\alpha_{k}-\alpha_{k-1}\right) s^{n-1}\right. \\
&\left.+K_{v}\left(t, s, s^{n-1} \alpha_{k-1}\right)\left(\alpha_{k}-\alpha_{k-1}\right) s^{n-1}\right] d s \\
&= f\left(t, t^{n-1} \alpha_{k}\right)+\int_{0}^{t} K\left(t, s, s^{n-1} \alpha_{k}\right) d s \\
&= F\left(t, t^{n-1} \alpha_{k} ; \alpha_{k}\right)+\int_{0}^{t} K^{*}\left(t, s, s^{n-1} \alpha_{k} ; \alpha_{k}\right) d s, \\
& \alpha_{k}(1) \leq g\left(\alpha_{k}\right)=h_{1}\left(\alpha_{k}, \alpha_{k} ; \beta_{0}\right) .
\end{aligned}
$$

Since $f_{v}(t, v), k_{v}(t, s, v)$ are increasing in $v$ by assumptions $\left(\mathrm{A}_{2}\right)$ and $\left(\mathrm{A}_{3}\right)$, therefore, $f_{v}\left(t, t^{n-1} \alpha_{0}\right) \leq f_{v}\left(t, t^{n-1} \alpha_{k}\right), K_{v}\left(t, s, s^{n-1} \alpha_{0}\right) \leq K_{v}\left(t, s, s^{n-1} \alpha_{k}\right)$ for $t^{n-1} \alpha_{0} \leq t^{n-1} \alpha_{k}$. Consequently, in view of (3.1), (3.2), (3.3), we obtain the inequality

$$
\begin{aligned}
-\left(t^{n} \beta_{k}^{\prime}\right)^{\prime}= & \bar{F}\left(t, t^{n-1} \beta_{k} ; \alpha_{0}, \beta_{k-1}\right)+\int_{0}^{t} K^{* *}\left(t, s, s^{n-1} \beta_{k} ; \alpha_{0}, \beta_{k-1}\right) d s \\
= & f\left(t, t^{n-1} \beta_{k-1}\right)+f_{v}\left(t, t^{n-1} \alpha_{0}\right)\left(\beta_{k}-\beta_{k-1}\right) t^{n-1} \\
& +\int_{0}^{t}\left[K\left(t, s, s^{n-1} \beta_{k-1}\right)+K_{v}\left(t, s, s^{n-1} \alpha_{0}\right)\left(\beta_{k}-\beta_{k-1}\right) s^{n-1}\right] d s \\
\geq & f\left(t, t^{n-1} \alpha_{k}\right)+f_{v}\left(t, t^{n-1} \alpha_{k}\right)\left(\beta_{k-1}-\alpha_{k}\right) t^{n-1}-f_{v}\left(t, t^{n-1} \alpha_{0}\right)\left(\beta_{k-1}-\beta_{k}\right) t^{n-1} \\
& +\int_{0}^{t}\left[K\left(t, s, s^{n-1} \alpha_{k}\right)+K_{v}\left(t, s, s^{n-1} \alpha_{k}\right)\left(\beta_{k-1}-\alpha_{k}\right) s^{n-1}\right. \\
& \left.-K_{v}\left(t, s, s^{n-1} \alpha_{0}\right)\left(\beta_{k-1}-\beta_{k}\right) s^{n-1}\right] d s \\
\geq & f\left(t, t^{n-1} \alpha_{k}\right)+f_{v}\left(t, t^{n-1} \alpha_{k}\right)\left(\beta_{k-1}-\alpha_{k}\right) t^{n-1}-f_{v}\left(t, t^{n-1} \alpha_{k}\right)\left(\beta_{k-1}-\beta_{k}\right) t^{n-1} \\
& +\int_{0}^{t}\left[K\left(t, s, s^{n-1} \alpha_{k}\right)+K_{v}\left(t, s, s^{n-1} \alpha_{k}\right)\left(\beta_{k-1}-\alpha_{k}\right) s^{n-1}\right. \\
& \left.-K_{v}\left(t, s, s^{n-1} \alpha_{k}\right)\left(\beta_{k-1}-\beta_{k}\right) s^{n-1}\right] d s \\
= & f\left(t, t^{n-1} \alpha_{k}\right)+f_{v}\left(t, t^{n-1} \alpha_{k}\right)\left(\beta_{k}-\alpha_{k}\right) t^{n-1}
\end{aligned}
$$




$$
\begin{aligned}
& +\int_{0}^{t}\left[K\left(t, s, s^{n-1} \alpha_{k}\right)+K_{v}\left(t, s, s^{n-1} \alpha_{k}\right)\left(\beta_{k}-\alpha_{k}\right) s^{n-1}\right] d s \\
= & F\left(t, t^{n-1} \beta_{k} ; \alpha_{k}\right)+\int_{0}^{t} K^{*}\left(t, s, s^{n-1} \beta_{k} ; \alpha_{k}\right) d s,
\end{aligned}
$$

and by virtue of the inequality

$$
\begin{aligned}
g\left(\beta_{k}\right)-h_{1}\left(\beta_{k}, \alpha_{k} ; \beta_{0}\right) \\
\quad=g\left(\beta_{k}\right)-g\left(\alpha_{k}\right)-g^{\prime}\left(\beta_{0}\right)\left(\beta_{k}-\alpha_{k}\right) \\
\quad=\left[g^{\prime}\left(c_{2}\right)-g^{\prime}\left(\beta_{0}\right)\right]\left(\beta_{k}-\alpha_{k}\right) \geq 0, \quad \alpha_{k} \leq c_{2} \leq \beta_{k} \leq \beta_{0},
\end{aligned}
$$

we get $\beta_{k}(1) \geq g\left(\beta_{k}\right)=h_{1}\left(\beta_{k}, \alpha_{k} ; \beta_{0}\right)$. Thus, as argued earlier, there exists a unique solution $\alpha_{k+1}$ of (3.6) such that

$$
t^{n-1} \alpha_{k}(t) \leq t^{n-1} \alpha_{k+1}(t) \leq t^{n-1} \beta_{k}(t), \quad t \in[0,1] .
$$

Now let us consider the following SBVP:

$$
\left\{\begin{aligned}
&-\left(t^{n} \beta_{k+1}^{\prime}\right)^{\prime}= \bar{F}\left(t, t^{n-1} \beta_{k+1} ; \alpha_{0}, \beta_{k}\right) \\
& \quad+\int_{0}^{t} K^{* *}\left(t, s, s^{n-1} \beta_{k+1} ; \alpha_{0}, \beta_{k}\right) d s, \quad 0<t<1, \\
& \lim _{t \rightarrow 0^{+}} t^{n-1} \beta_{k+1}(t)=u_{0}, \quad \beta_{k+1}(1)=h_{2}\left(\beta_{k+1}, \beta_{k}\right) .
\end{aligned}\right.
$$

Again, using assumptions $\left(A_{2}\right)-\left(A_{4}\right),(3.1),(3.2),(3.3)$, and the inequality

$$
t^{n-1} \alpha_{0} \leq t^{n-1} \alpha_{k-1} \leq t^{n-1} \alpha_{k} \leq t^{n-1} \beta_{k} \leq t^{n-1} \beta_{k-1} \leq t^{n-1} \beta_{0},
$$

we obtain

$$
\begin{aligned}
-\left(t^{n} \beta_{k}^{\prime}\right)^{\prime}= & \bar{F}\left(t, t^{n-1} \beta_{k} ; \alpha_{0}, \beta_{k-1}\right)+\int_{0}^{t} K^{* *}\left(t, s, s^{n-1} \beta_{k} ; \alpha_{0}, \beta_{k-1}\right) d s \\
= & f\left(t, t^{n-1} \beta_{k-1}\right)+f_{v}\left(t, t^{n-1} \alpha_{0}\right)\left(\beta_{k}-\beta_{k-1}\right) t^{n-1} \\
& +\int_{0}^{t}\left[K\left(t, s, s^{n-1} \beta_{k-1}\right)+K_{v}\left(t, s, s^{n-1} \alpha_{0}\right)\left(\beta_{k}-\beta_{k-1}\right) s^{n-1}\right] d s \\
\geq & f\left(t, t^{n-1} \beta_{k}\right)+f_{v}\left(t, t^{n-1} \beta_{k}\right)\left(\beta_{k-1}-\beta_{k}\right) t^{n-1} \\
& -f_{v}\left(t, t^{n-1} \alpha_{0}\right)\left(\beta_{k-1}-\beta_{k}\right) t^{n-1} \\
& +\int_{0}^{t}\left[K\left(t, s, s^{n-1} \beta_{k}\right)+K_{v}\left(t, s, s^{n-1} \beta_{k}\right)\left(\beta_{k-1}-\beta_{k}\right) s^{n-1}\right. \\
& \left.-K_{v}\left(t, s, s^{n-1} \alpha_{0}\right)\left(\beta_{k-1}-\beta_{k}\right) s^{n-1}\right] d s \\
\geq & f\left(t, t^{n-1} \beta_{k}\right)+\int_{0}^{t} K\left(t, s, s^{n-1} \beta_{k}\right) d s \\
= & \bar{F}\left(t, t^{n-1} \beta_{k} ; \alpha_{0}, \beta_{k}\right)+\int_{0}^{t} K^{* *}\left(t, s, s^{n-1} \beta_{k} ; \alpha_{0}, \beta_{k}\right) d s, \\
& \beta_{k}(1) \geq g\left(\beta_{k}\right)=h_{2}\left(\beta_{k}, \beta_{k}\right),
\end{aligned}
$$




$$
\begin{aligned}
-\left(t^{n} \alpha_{k}^{\prime}\right)^{\prime}= & F\left(t, t^{n-1} \alpha_{k} ; \alpha_{k-1}\right)+\int_{0}^{t} K^{*}\left(t, s, s^{n-1} \alpha_{k} ; \alpha_{k-1}\right) d s \\
= & f\left(t, t^{n-1} \alpha_{k-1}\right)+f_{v}\left(t, t^{n-1} \alpha_{k-1}\right)\left(\alpha_{k}-\alpha_{k-1}\right) t^{n-1} \\
& +\int_{0}^{t}\left[K\left(t, s, s^{n-1} \alpha_{k-1}\right)+K_{v}\left(t, s, s^{n-1} \alpha_{k-1}\right)\left(\alpha_{k}-\alpha_{k-1}\right) s^{n-1}\right] d s \\
\leq & f\left(t, t^{n-1} \beta_{k}\right)-f_{v}\left(t, t^{n-1} \alpha_{k-1}\right)\left(\beta_{k}-\alpha_{k-1}\right) t^{n-1}+f_{v}\left(t, t^{n-1} \alpha_{k-1}\right)\left(\alpha_{k}-\alpha_{k-1}\right) t^{n-1} \\
& +\int_{0}^{t}\left[K\left(t, s, s^{n-1} \beta_{k}\right)-K_{v}\left(t, s, s^{n-1} \alpha_{k-1}\right)\left(\beta_{k}-\alpha_{k-1}\right) s^{n-1}\right. \\
& \left.+K_{v}\left(t, s, s^{n-1} \alpha_{k-1}\right)\left(\alpha_{k}-\alpha_{k-1}\right) s^{n-1}\right] d s \\
= & f\left(t, t^{n-1} \beta_{k}\right)-f_{v}\left(t, t^{n-1} \alpha_{k-1}\right)\left(\beta_{k}-\alpha_{k}\right) t^{n-1} \\
& +\int_{0}^{t}\left[K\left(t, s, s^{n-1} \beta_{k}\right)-K_{v}\left(t, s, s^{n-1} \alpha_{k-1}\right)\left(\beta_{k}-\alpha_{k}\right) s^{n-1}\right] d s \\
\leq & f\left(t, t^{n-1} \beta_{k}\right)+f_{v}\left(t, t^{n-1} \alpha_{0}\right)\left(\alpha_{k}-\beta_{k}\right) t^{n-1} \\
& +\int_{0}^{t}\left[K\left(t, s, s^{n-1} \beta_{k}\right)+K_{v}\left(t, s, s^{n-1} \alpha_{0}\right)\left(\alpha_{k}-\beta_{k}\right) s^{n-1}\right] d s \\
= & \bar{F}\left(t, t^{n-1} \alpha_{k} ; \alpha_{0}, \beta_{k}\right)+\int_{0}^{t} K^{* *}\left(t, s, s^{n-1} \alpha_{k} ; \alpha_{0}, \beta_{k}\right) d s, \\
& \alpha_{k}(1)=g\left(\alpha_{k}\right) \leq h_{2}\left(\alpha_{k}, \alpha_{k-1}\right) .
\end{aligned}
$$

Applying the earlier arguments, it follows that there exists a unique solution $\beta_{k+1}$ of (3.7) such that

$$
t^{n-1} \alpha_{k}(t) \leq t^{n-1} \beta_{k+1}(t) \leq t^{n-1} \beta_{k}(t), \quad t \in[0,1]
$$

Following the procedure employed to prove $t^{n-1} \alpha_{1}(t) \leq t^{n-1} \beta_{1}(t)$, it can be shown that $t^{n-1} \alpha_{k+1}(t) \leq t^{n-1} \beta_{k+1}(t)$. Hence we have

$$
\begin{aligned}
t^{n-1} \alpha_{0}(t) & \leq t^{n-1} \alpha_{1}(t) \leq \cdots \leq t^{n-1} \alpha_{k+1}(t) \leq t^{n-1} \beta_{k+1}(t) \\
& \leq \cdots \leq t^{n-1} \beta_{1}(t) \leq t^{n-1} \beta_{0}(t), \quad t \in[0,1] .
\end{aligned}
$$

As the monotone sequences $\left\{t^{n-1} \alpha_{k+1}(t)\right\}$ and $\left\{t^{n-1} \beta_{k+1}(t)\right\}$ are both bounded, therefore, they converge to the limit functions $t^{n-1} \rho(t)$ and $t^{n-1} \sigma(t)$ pointwise respectively.

Now we show that the convergence of the sequences $\left\{t^{n-1} \alpha_{k+1}(t)\right\}$ and $\left\{t^{n-1} \beta_{k+1}(t)\right\}$ to the limit functions $\left\{t^{n-1} \rho(t)\right\}$ and $\left\{t^{n-1} \sigma(t)\right\}$ respectively is indeed uniform. Using SBVP (3.6), integral equation (2.1), and relation (3.8), we observe that $\left\{t^{n-1} \alpha_{k+1}(t)\right\},\left\{\left(t^{n-1} \alpha_{k+1}(t)\right)^{\prime}\right\}$, $\left\{t^{n} \alpha_{k+1}^{\prime}(t)\right\},\left\{\left(t^{n} \alpha_{k+1}^{\prime}(t)\right)^{\prime}\right\}$ are uniformly bounded sequences. Thus, by the Arzela-Ascoli theorem, the sequences $\left\{t^{n-1} \alpha_{k+1}(t)\right\},\left\{t^{n} \alpha_{k+1}^{\prime}(t)\right\}$ have uniformly convergent subsequences. Hence, by the monotonicity of the sequence $\left\{t^{n-1} \alpha_{k+1}(t)\right\}$ in $\mathcal{E}$, it follows that the sequence converges uniformly to the limit function $t^{n-1} \rho(t)$ in $\mathcal{E}$. In a similar manner, the sequence $\left\{t^{n-1} \beta_{k+1}(t)\right\}$ in $\mathcal{E}$ converges uniformly to the limit function $t^{n-1} \sigma(t)$ in $\mathcal{E}$.

Finally, we show that the convergence of the sequences is quadratic. We only prove the quadratic convergence for the sequence $\left\{t^{n-1} \alpha_{k+1}(t)\right\}$ as that of the sequence $\left\{t^{n-1} \beta_{k+1}(t)\right\}$ follows a similar procedure. 
Let us define

$$
e_{k+1}(t)=\rho(t)-\alpha_{k+1}(t)
$$

Then, for $t^{n-1} \zeta_{1} \in\left(t^{n-1} \alpha_{k}, t^{n-1} \rho\right), s^{n-1} \zeta_{2} \in\left(s^{n-1} \alpha_{k}, s^{n-1} \rho\right), t^{n-1} \zeta_{3} \in\left(t^{n-1} \alpha_{k}, t^{n-1} \zeta_{1}\right), s^{n-1} \zeta_{4} \in$ $\left(s^{n-1} \alpha_{k}, s^{n-1} \zeta_{2}\right)$, we have

$$
\begin{aligned}
-( & \left.t^{n} \alpha_{k+1}^{\prime}(t)\right)^{\prime} \\
= & -\left(t^{n} \rho^{\prime}(t)\right)^{\prime}+\left(t^{n} \alpha_{k+1}^{\prime}(t)\right)^{\prime} \\
= & f\left(t, t^{n-1} \rho(t)\right)+\int_{0}^{t} K\left(t, s, s^{n-1} \rho(s)\right) d s \\
& -f\left(t, t^{n-1} \alpha_{k}\right)-f_{v}\left(t, t^{n-1} \alpha_{k}\right)\left(\alpha_{k+1}-\alpha_{k}\right) t^{n-1} \\
& -\int_{0}^{t}\left[K\left(t, s, s^{n-1} \alpha_{k}\right)+K_{v}\left(t, s, s^{n-1} \alpha_{k}\right)\left(\alpha_{k+1}-\alpha_{k}\right) s^{n-1}\right] d s \\
= & f_{v}\left(t, t^{n-1} \zeta_{1}\right)\left(\rho-\alpha_{k}\right) t^{n-1}-f_{v}\left(t, t^{n-1} \alpha_{k}\right)\left(\alpha_{k+1}-\alpha_{k}\right) t^{n-1} \\
& +\int_{0}^{t}\left[K_{v}\left(t, s, s^{n-1} \zeta_{2}\right)\left(\rho-\alpha_{k}\right) s^{n-1}-K_{v}\left(t, s, s^{n-1} \alpha_{k}\right)\left(\alpha_{k+1}-\alpha_{k}\right) s^{n-1}\right] d s \\
= & {\left[f_{v}\left(t, t^{n-1} \zeta_{1}\right)-f_{v}\left(t, t^{n-1} \alpha_{k}\right)\right]\left(\rho-\alpha_{k}\right) t^{n-1}+f_{v}\left(t, t^{n-1} \alpha_{k}\right)\left(\rho-\alpha_{k+1}\right) t^{n-1} } \\
& +\int_{0}^{t}\left[\left\{K_{v}\left(t, s, s^{n-1} \zeta_{2}\right)-K_{v}\left(t, s, s^{n-1} \alpha_{k}\right)\right\}\left(\rho-\alpha_{k}\right) s^{n-1}\right. \\
& \left.+K_{v}\left(t, s, s^{n-1} \alpha_{k}\right)\left(\rho-\alpha_{k+1}\right) s^{n-1}\right] d s \\
= & f_{v v}\left(t, t^{n-1} \zeta_{3}\right)\left(\zeta_{1}-\alpha_{k}\right)\left(\rho-\alpha_{k}\right)\left(t^{n-1}\right)^{2}+f_{v}\left(t, t^{n-1} \alpha_{k}\right)\left(\rho-\alpha_{k+1}\right) t^{n-1} \\
& +\int_{0}^{t}\left[K_{v v}\left(t, s, s^{n-1} \zeta_{4}\right)\left(\zeta_{2}-\alpha_{k}\right)\left(\rho-\alpha_{k}\right)\left(s^{n-1}\right)^{2}\right. \\
& \left.+K_{v}\left(t, s, s^{n-1} \alpha_{k}\right)\left(\rho-\alpha_{k+1}\right) s^{n-1}\right] d s \\
\leq & f_{v v}\left(t, t^{n-1} \zeta_{3}\right)\left(\left(\rho-\alpha_{k}\right) t^{n-1}\right)^{2}+f_{v}\left(t, t^{n-1} \alpha_{k}\right)\left(\rho-\alpha_{k+1}\right) t^{n-1} \\
& +\int_{0}^{t}\left[K_{v v}\left(t, s, s^{n-1} \zeta_{4}\right)\left(\left(\rho-\alpha_{k}\right) s^{n-1}\right)^{2}+K_{v}\left(t, s, s^{n-1} \alpha_{k}\right)\left(\rho-\alpha_{k+1}\right) s^{n-1}\right] d s .
\end{aligned}
$$

Setting $N=N_{1}+N_{2}, M=M_{1}+M_{2}$, where $N_{1}$ and $N_{2}$ provide bounds for $f_{v v}(t, \cdot)\left(t^{n-1}\right)^{2}$ and $\int_{0}^{t}\left[K_{v v}(t, s, \cdot)\left(s^{n-1}\right)^{2}\right] d s$ respectively and $f_{v}(t, \cdot) \leq-M_{1}, \int_{0}^{t} K_{v}(t, s, \cdot) d s \leq-M_{2}$, the above inequality takes the form

$$
-\left(t^{n} \alpha_{k+1}^{\prime}(t)\right)^{\prime} \leq N\left|e_{k}\right|^{2}-M e_{k+1(t)} t^{n-1}
$$

Given $M, N$ and $\left|e_{k}\right|^{2}$, there exists $\mu \in \mathbb{R}^{+}$such that

$$
0=N\left|e_{k}\right|^{2}-M \mu t^{n-1}
$$

By a comparison theorem [56], it follows from (3.9) and (3.10) that

$$
e_{k+1}(t) t^{n-1} \leq \mu t^{n-1}=\frac{N}{M}\left|e_{k}\right|^{2}, \quad t \in[0,1),
$$


which implies that

$$
\left|e_{k+1}\right| \leq \frac{N}{M}\left|e_{k}\right|^{2}
$$

Now, we consider

$$
\begin{aligned}
e_{k+1}(1) & =\rho(1)-\alpha_{k+1}(1) \\
& =g(\rho)-h_{1}\left(\alpha_{k+1}, \alpha_{k}, \beta_{0}\right) \\
& =g(\rho)-g\left(\alpha_{k}\right)-g^{\prime}\left(\beta_{0}\right)\left(\alpha_{k+1}-\alpha_{k}\right) \\
& =\left[g^{\prime}\left(\eta_{1}\right)-g^{\prime}\left(\beta_{0}\right)\right]\left(\rho-\alpha_{k}\right)+g^{\prime}\left(\beta_{0}\right)\left(\rho-\alpha_{k+1}\right) \\
& \leq\left[g^{\prime}\left(\alpha_{k}\right)-g^{\prime}(\rho)\right]\left(\rho-\alpha_{k}\right)+g^{\prime}\left(\beta_{0}\right)\left(\rho-\alpha_{k+1}\right) \\
& =-g^{\prime \prime}\left(\eta_{2}\right)\left|e_{k}\right|^{2}+g^{\prime}\left(\beta_{0}\right) e_{k+1},
\end{aligned}
$$

where $\eta_{1}, \eta_{2} \in\left(\alpha_{k}, \rho\right)$. Letting $g^{\prime \prime}(\cdot) \leq-\lambda_{2}$ on $\left(\alpha_{k}, \rho\right)$ and $g^{\prime}(\cdot) \leq \lambda_{1}<1$, we find that

$$
\left|e_{k+1}\right| \leq \frac{\lambda_{2}}{1-\lambda_{1}}\left|e_{k}\right|^{2}
$$

From (3.11) and (3.12), we conclude that the sequence $\left\{t^{n-1} \alpha_{k+1}(t)\right\}$ converges to the unique solution of SBVP (1.1) quadratically. Similarly, we can prove the quadratic convergence of the sequence $\left\{t^{n-1} \beta_{k+1}(t)\right\}$. This completes the proof.

Example Consider the following singular nonlocal boundary value problem:

$$
\left\{\begin{aligned}
-\left(t^{3} u^{\prime}\right)^{\prime}= & A(1+t) e^{-t^{2} u(t)+1} \\
& +\int_{0}^{t}\left[B(2-t) s^{2} e^{-s^{2} u(s)-1}-C s^{2} u(s)\right] d s, \quad 0<t<1, \\
\lim _{t \rightarrow 0^{+}} & t^{2} u(t)=0, \quad u(1)=1+\frac{1}{2} u\left(\frac{1}{2}\right),
\end{aligned}\right.
$$

where $f\left(t, t^{2} u(t)\right)=A(1+t) e^{-t^{2} u(t)+1}, K(t, s, u(s))=\left[B(2-t) s^{2} e^{-s^{2} u(s)-1}-C s^{2} u(s)\right], n=3$, $g(u(1 / 2))=1+\frac{1}{2} u\left(\frac{1}{2}\right)$, and $A, B, C$ are suitable positive constants. Let $\alpha(t)=0$ and $\beta(t)=2 t$ be respectively lower and upper solutions of (3.13). Clearly, $\alpha(t)$ and $\beta(t)$ are not the solutions of (3.13) and $\alpha(t) \leq \beta(t), t \in[0,1]$. Moreover, assumptions $\left(\mathrm{A}_{1}\right),\left(\mathrm{A}_{2}\right),\left(\mathrm{A}_{3}\right)$, and $\left(\mathrm{A}_{4}\right)$ of Theorem 3.1 are satisfied. Thus, the conclusion of Theorem 3.1 applies to problem (3.13).

\section{Conclusions}

In this paper, we have presented monotone sequences of approximate solutions converging uniformly and quadratically to a unique solution of a nonlocal singular boundary value problem involving second-order integro-differential equations in weighted spaces. The results established in this project are new and contribute to the present theory of singular boundary value problems of integro-differential equations. The present work provides a guideline to extend it further by relaxing the convexity assumptions on the nonlinear functions $f(t, v)$ and $K(t, s, v)$ in (1.1). In fact, we can find a continuous function $\phi(t, v)$ such that $f_{v v}(t, v)+\phi_{v v}(t, v) \geq 0$, where $\phi_{v v}(t, v)$ exists, continuous with $\phi_{v v}(t, v) \geq 0$. In a similar manner, the convexity assumption on $K(t, s, v)$ and the concavity assumption on $g(u)$ can be 
relaxed. Further, for $g(u)=u_{1}$ and $K(t, s, v) \equiv 0$ in (1.1), our results become the existence results obtained in [49]. The results for a nonlocal singular boundary value problem of second-order integro-differential equations involving a purely integral type of nonlinearity follow by taking $f(t, v) \equiv 0$ in (1.1). Thus, the work presented in this paper takes care of numerous interesting situations.

\section{Competing interests}

The authors declare that they have no competing interests.

Authors' contributions

Each of the authors, RPA, BA and AA contributed to each part of this work equally and read and approved the final version of the manuscript.

\section{Author details}

${ }^{1}$ Department of Mathematics, Texas A\&M University, Kingsville, 78363-8202, USA. ${ }^{2}$ Department of Mathematics, Faculty of Science, King Abdulaziz University, P.O. Box 80203, Jeddah, 21589, Saudi Arabia.

\section{Acknowledgements}

This work was supported by Deanship of Scientific Research (DSR), King Abdulaziz University, Jeddah, Saudi Arabia.

\section{Received: 30 September 2013 Accepted: 4 November 2013 Published: 27 Nov 2013}

\section{References}

1. Castro, A, Kurepa, A: Energy analysis of a nonlinear singular equation and applications. Rev. Colomb. Mat. 21, 155-166 (1988)

2. Kurepa, A: Existence and uniqueness theorem for singular initial value problem and applications. Publ. Inst. Math. (Belgr.) 45, 89-93 (1990)

3. Agarwal, RP, O'Regan, D: Infinite interval problems modeling phenomena which arise in the theory of plasma and electrical potential theory. Stud. Appl. Math. 111, 339-358 (2003)

4. Shin, JY: A singular nonlinear boundary value problem in the nonlinear circular membrane under normal pressure. J. Korean Math. Soc. 32, 761-773 (1995)

5. Baxley, J: A singular nonlinear boundary value problem: membrane response of a spherical cap. SIAM J. Appl. Math. 48, 497-505 (1988)

6. Agarwal, RP, O'Regan, D, Lakshmikantham, V: Existence criteria for singular boundary value problems modelling the membrane response of a spherical cap. Nonlinear Anal., Real World Appl. 4, 223-244 (2003)

7. Johnson, KN: Circularly symmetric deformations of shallow elastic membrane caps. Q. Appl. Math. 55, 537-550 (1997)

8. Agarwal, RP, O'Regan, D: Boundary value problems on the half line in the theory of colloids. Math. Probl. Eng. 8, 143-150 (2002)

9. Soewona, E, Vajravelu, K, Mohapatra, RN: Existence of solutions of a nonlinear boundary value problem arising in the flow and heat transfer over a stretching sheet. Nonlinear Anal. 18, 93-98 (1992)

10. Bernis, F, Peletier, LA: Two problems from draining flows involving third order ordinary differential equations. SIAM J. Math. Anal. 27, 515-527 (1996)

11. Wang, JY, Zhang, ZX: A boundary value problem from draining and coating flows involving a third order differential equation. Z. Angew. Math. Phys. 49, 506-513 (1998)

12. Agarwal, RP, O'Regan, D: Infinite interval problems modeling the flow of a gas through a semi-infinite porous medium. Stud. Appl. Math. 108, 245-257 (2002)

13. Agarwal, RP, O'Regan, D: A singular Homann differential equation. Z. Angew. Math. Mech. 83, 344-350 (2003)

14. Wang, J, Gao, W, Zhang, Z: Singular nonlinear boundary value problems arising in boundary layer theory. J. Math. Anal. Appl. 233, 246-256 (1999)

15. Nachman, A, Callegari, A: A nonlinear singular boundary value problem in the theory of pseudoplastic fluids. SIAM J. Appl. Math. 38, 275-282 (1980)

16. O'Regan, D: Theory of Singular Boundary Value Problems. World Scientific, Singapore (1994)

17. O'Regan, D: Existence Theory for Nonlinear Ordinary Differential Equations. Kluwer Academic, Dordrecht (1997)

18. Kiguradze, IT, Lomtatidze, AG: On certain boundary value problems for second-order linear ordinary differential equations with singularities. J. Math. Anal. Appl. 101, 325-347 (1984)

19. Lomtatidze, AG: A boundary value problem for second-order nonlinear ordinary differential equations with singularities. Differ. Equ. 22, 416-426 (1986)

20. Lomtatidze, AG: Positive solutions of boundary value problems for second-order ordinary differential equations with singularities. Differ. Equ. 23, 1146-1152 (1987)

21. Agarwal, RP, O'Regan, D: Positive solutions to superlinear singular boundary value problems. J. Comput. Appl. Math. 88, 129-147 (1998)

22. O'Regan, D: Singular second order boundary value problems. Nonlinear Anal. 15, 1097-1109 (2000)

23. Hao, Z-C, Liang, J, Xiao, T-J: Positive solutions of operator equations on half-line. J. Math. Anal. Appl. 314, 423-435 (2006)

24. Liu, L, Zhang, X, Wu, Y: Existence of positive solutions for singular higher-order differential equations. Nonlinear Anal. 68, 3948-3961 (2008)

25. Wang, Y, Liu, L, Wu, Y: Positive solutions of singular boundary value problems on the half-line. Appl. Math. Comput. $197,789-796(2008)$ 
26. Ladde, GS, Lakshmikantham, V, Vatsala, AS: Monotone Iterative Techniques for Nonlinear Differential Equations. Pitman, Boston (1985)

27. Bellman, R, Kalaba, R: Quasilinearization and Nonlinear Boundary Value Problems. Am. Elsevier, New York (1965)

28. Lakshmikantham, V, Vatsala, AS: Generalized Quasilinearization for Nonlinear Problems. Mathematics and Its Applications, vol. 440. Kluwer Academic, Dordrecht (1998)

29. Mandelzweig, VB, Tabakin, F: Quasilinearization approach to nonlinear problems in physics with application to nonlinear ODEs. Comput. Phys. Commun. 141, 268-281 (2001)

30. Eloe, $\mathrm{P}, \mathrm{Gao}, \mathrm{Y}$ : The method of quasilinearization and a three-point boundary value problem. J. Korean Math. Soc. 39 319-330 (2002)

31. Cabada, A, Nieto, J: Quasilinearization and rate of convergence for higher order nonlinear periodic boundary value problems. J. Optim. Theory Appl. 108, 97-107 (2001)

32. Nikolov, S, Stoytchev, S, Torres, A, Nieto, Jj: Biomathematical modeling and analysis of blood flow in an intracranial aneurysms. Neurol. Res. 25, 497-504 (2003)

33. Ahmad, B: A quasilinearization method for a class of integro-differential equations with mixed nonlinearities. Nonlinear Anal., Real World Appl. 7, 997-1004 (2006)

34. Krivec, R, Mandelzweig, VB: Quasilinearization method and WKB. Comput. Phys. Commun. 174, 119-126 (2006)

35. Akyildiz, FT, Vajravelu, K: Existence, uniqueness, and quasilinearization results for nonlinear differential equations arising in viscoelastic fluid flow. Differ. Equ. Nonlinear Mech. 2006, Article ID 71717 (2006)

36. Amster, P, De Napoli, P: A quasilinearization method for elliptic problems with a nonlinear boundary condition. Nonlinear Anal. 66, 2255-2263 (2007)

37. Pei, M, Chang, SK: A quasilinearization method for second-order four-point boundary value problems. Appl. Math. Comput. 202, 54-66 (2008)

38. Pei, M, Chang, SK: A quasilinearization method for second-order four-point boundary value problems. Appl. Math. Comput. 202, 54-66 (2008)

39. Ahmad, B, Nieto, J: Existence and approximation of solutions for a class of nonlinear impulsive functional differential equations with anti-periodic boundary conditions. Nonlinear Anal. 69, 3291-3298 (2008)

40. Ahmad, B, Alghamdi, B: Approximation of solutions of the nonlinear Duffing equation involving both integral and non-integral forcing terms with separated boundary conditions. Comput. Phys. Commun. 179, 409-416 (2008)

41. Ahmad, B, Alsaedi, A: Existence of approximate solutions of the forced Duffing equation with discontinuous type integral boundary conditions. Nonlinear Anal., Real World Appl. 10, 358-367 (2009)

42. Nieto, JJ, Ahmad, B: Approximation of solutions for an initial and terminal value problem for the forced Duffing equation with non-viscous damping. Appl. Math. Comput. 216, 2129-2136 (2010)

43. Sun, L, Zhou, M, Wang, G: Generalized quasilinearization method for nonlinear boundary value problems with integral boundary conditions. Electron. J. Qual. Theory Differ. Equ. 66, 1-14 (2010)

44. Alsaedi, A: Approximation of solutions for second-order $m$-point nonlocal boundary value problems via the method of generalized quasilinearization. Bound. Value Probl. 2011, Article ID 929061 (2011)

45. Wang, P, Gao, W: Quasilinearization of an initial value problem for a set valued integro-differential equation. Comput. Math. Appl. 61, 2111-2115 (2011)

46. Motsa, SS: A new piecewise-quasilinearization method for solving chaotic systems of initial value problems. Cent. Eur. J. Phys. 10, 936-946 (2012)

47. Hartung, F: Parameter estimation by quasilinearization in differential equations with state-dependent delays. Discrete Contin. Dyn. Syst., Ser. B 18, 1611-1631 (2013)

48. Motsa, SS, Sibanda, P: Some modifications of the quasilinearization method with higher-order convergence for solving nonlinear BVPs. Numer. Algorithms 63, 399-417 (2013)

49. Devi, JV, Vatsala, AS: Quasilinearization for second order singular boundary value problems with solutions in weighted spaces. J. Korean Math. Soc. 37, 823-832 (2000)

50. Eloe, PW: The quasilinearization method on an unbounded domain. Proc. Am. Math. Soc. 131, 1481-1488 (2002)

51. Ramos, Jl: Piecewise quasilinearization techniques for singular boundary-value problems. Comput. Phys. Commun. $158,12-25(2004)$

52. El-Gebeily, M, O'Regan, D: A quasilinearization method for a class of second order singular nonlinear differential equations with nonlinear boundary conditions. Nonlinear Anal., Real World Appl. 8, 174-186 (2007)

53. Kannan, R, O'Regan, D: A note on singular boundary value problems with solutions in weighted spaces. Nonlinear Anal. 37, 791-796 (1999)

54. Agarwal, RP, O'Regan, D, Lakshmikantham, V, Leela, S: Existence of positive solutions for singular initial and boundary value problems via the classical upper and lower solution approach. Nonlinear Anal. 50, 215-222 (2002)

55. Lakshmikantham, V, Rao, MRM: Theory of Integro-Differential Equations. Gordon \& Breach, London (1995)

56. Lakshmikantham, V, Leela, S: Differential and Integral Inequalities. vol. I. Academic Press, New York (1969)

10.1186/1687-2770-2013-261

Cite this article as: Agarwal et al.: Method of quasilinearization for a nonlocal singular boundary value problem in weighted spaces. Boundary Value Problems 2013, 2013:261 